\title{
Design and engineering of metasurfaces for high-directivity antenna and sensing applications
}

\author{
Badreddine Ratni ${ }^{1, a}$, Elena Bochkova ${ }^{1, a}$, Gérard-Pascal Piau ${ }^{2}$, André de Lustrac ${ }^{1,3}$, Anatole Lupu ${ }^{1}$ \\ and Shah Nawaz Burokur ${ }^{4, *}$ \\ ${ }^{1}$ Centre de Nanosciences et de Nanotechnologies, CNRS, Univ Paris Sud, Université Paris-Saclay, C2N - Orsay, 91405 Orsay, France \\ 2 AIRBUS Group Innovations, 92150 Suresnes, France \\ 3 Université Paris Ouest, 92410 Ville d'Avray, France \\ 4 LEME, EA 4416, Université Paris Ouest, 92410 Ville d'Avray, France
}

Received 30 March 2016 / Accepted 19 June 2016

\begin{abstract}
The engineering of metasurfaces for antenna and sensing applications is investigated. The metasurfaces composed of $L C$-resonant cells, are designed to achieve either modulated phase or high quality factor resonances. While the phase modulated metasurface applied as a superstrate in a Fabry-Perot cavity antenna, shows the capability to steer the radiated beam to an off-normal radiation, the high quality factor resonant metasurface presents high sensitivity to the presence of substances.
\end{abstract}

Key words: Metasurfaces, Resonance, Antennas, Sensing.

\section{Introduction}

Metamaterial surfaces also known as metasurfaces have made relevant a wide array of interesting applications due to their unusual electromagnetic properties. For antenna applications in the microwave domain, they have been primarily used for filtering [1] and decoupling [2]. Metasurfaces embedded in parallel plate waveguides presenting gradient index have afterwards been applied as ultra-thin lenses to achieve high directivity [3, 4]. Refractive index is varied throughout the lens instead of relying on the interfaces of the dielectric material to control light flow. Other impedance modulated metasurfaces have also been proposed to transform surface or guided waves into radiated waves [5]. Metasurfaces have also been designed to act as partially reflective surfaces (PRS) in reflex-type Fabry-Perot (FP) cavity antennas in order to achieve high directivity from a single primary source [6-8].

In the field of resonance engineering, metasurfaces composed of generally two or three different resonators have been intensively studied to obtain an analogy of electromagnetically induced transparency (EIT) [9]. The concept of dark states was initially introduced in atomic physics. A dark state refers to a state of an atom or molecule that cannot absorb or emit photons. The concept has been intensively exploited during the last decade in the fields of photonics such as

\footnotetext{
${ }^{a}$ These authors contributed equally to the work.

*e-mail: sburokur@u-parislo.fr
}

integrated optics [10-12], photonic crystals [13, 14], metamaterials [15] or plasmonics [9, 16]. The interests to this domain were principally driven so as to obtain sharp spectral transmission features within an absorption band, which are highly desirable in sensing applications [15]. Most of studied designs in literature for EIT are based on the association of a superradiant element bearing an electric dipolar momentum and acting as a bright (or radiative) mode, with a subradiant element bearing an electric quadripolar or magnetic dipolar momentum and playing the role of the dark (or trapped) mode. For example, asymmetric coupled split-ring resonators [17-20], bilayer fish-scale [21] and cut wires structures $[22,23]$ have been used. The mode hybridization induced by the strong coupling between the directly excited bright and the indirectly excited dark elements then leads to a destructive Fano interference and thus to the opening of the narrow EIT window inside the absorption band. However, recent theoretical advances have shown that Fano resonances can be described by the interference of bright modes only and thus dark mode excitation is not necessary for their existence [24-26]. The term "dark mode" used in the context of this work is distinctly different from that used in association with the EIT effect. In our case, the term "dark mode" is used to specify eigenmodes with strongly reduced radiative damping resulting in a weak coupling to free space.

This present study deals with the design and engineering of $L C$-resonant metasurfaces for antenna and sensing 
applications. We propose firstly the design of phase modulated metasurface in order to steer an antenna's radiated beam. Phase modulation is achieved by judiciously varying the inductance of the metasurface. Secondly, we present the design of a metasurface where dark mode excitation is solely based on symmetry matching conditions of only one type of meta-atom rather than mode hybridization between two or more metaatoms. The design of the metasurfaces is made through numerical analysis using finite element method based commercial software HFSS from ANSYS [27]. Reported experimental measurements carried out on fabricated prototypes agree qualitatively with simulations.

\section{Metasurfaces for high directivity antennas}

\subsection{Phase modulated metasurface design}

The unit cell of the metasurface consists of simultaneously a capacitive and an inductive grid on the two faces of a Rogers TMM4 $\left(\varepsilon_{\mathrm{r}}=4.5\right.$ and $\left.\tan \delta=0.002\right)$ dielectric substrate of thickness $t=2.54 \mathrm{~mm}$, as presented in Figure 1a. The unit cell is chosen in order to have an $L C$ resonance allowing to produce a phase in reflection that varies from $+180^{\circ}$ to $-180^{\circ}$. The capacitive grid of the metasurface is formed by a row of metallic patches with width $w=27.2 \mathrm{~mm}$ and period $p=30 \mathrm{~mm}$, whereas the inductive grid consists of mesh strips having similar period $p$ and width $l$. The dimensions have been optimized in order to have a resonance around $2 \mathrm{GHz}$. Therefore by fixing $w$ and $p$, resonance frequency can be varied around $2 \mathrm{GHz}$. The PRS is composed of 20 by 20 cells $(600 \mathrm{~mm} \times 600 \mathrm{~mm})$, where each cell presents an elementary $L C$ resonance. Phase modulation along the metasurface can be achieved by varying the strip width $l$, which will allow engineering the desired gradient for controlling the direction of the radiated beam.

A parametric analysis is performed on the unit cell together with appropriate periodic boundary conditions. The results presented in Figure $1 \mathrm{~b}$ firstly show a resonance frequency lying between 1.6 and $2.23 \mathrm{GHz}$ for $l$ ranging from 4 to $20 \mathrm{~mm}$. The size of the different patterns has been chosen in order to have the phase of the reflection coefficient below $0^{\circ}$ near $2.16 \mathrm{GHz}$ while providing a sufficiently low transmittance (high reflectance). Figure 1c shows the variation of the transmission phase versus frequency. At a fixed frequency, a phase that varies with $l$ is obtained. With the geometrical dimensions used, a phase gradient of $85^{\circ}$ can be obtained at $2.16 \mathrm{GHz}$ in transmission, as illustrated in Figure 1d.

\subsection{Beam steering in high directivity antennas}

Metasurfaces with high reflectance have been proposed as superstrate reflectors in Fabry-Perot (FP) cavity antennas [6-8]. They are designed to act as partially reflective surfaces (PRS) in order to achieve high directivity from a single source. A cavity antenna is formed by a feeding source placed between two reflecting surfaces. The reflection phase value of the PRS is an important parameter since it allows to calculate the resonance frequency of the cavity. Therefore using low reflection phase values allows to design very thin cavity antennas. The resonance condition is given as:

$$
h+t \sqrt{\varepsilon_{\mathrm{r}}}=\left(\phi_{\mathrm{PRS}}+\phi_{\mathrm{r}}\right) \frac{\lambda}{4 \pi} \pm N \frac{\lambda}{2}
$$

where $\phi_{\mathrm{PRS}}$ is the reflection phase of the metasurface used as a PRS, $\phi_{\mathrm{r}}$ is the reflection phase of the reflector near the antenna (PEC in our case), and $N$ is an integer corresponding to the order of the cavity's electromagnetic mode. $t$ and $\varepsilon_{\mathrm{r}}$ are respectively the thickness and the relative permittivity of the antenna's dielectric board.

The cavity depictured in Figure $2 \mathrm{~b}$ is composed of a perfect electric conductor (PEC) surface acting as a conventional ground plane for the feeding source and the PRS. Furthermore, metasurfaces presenting a gradient in phase have been successfully used for steering the radiated beam in FP cavity antennas [28-33]. While a variation in capacitance was applied in references [28-31], a variation of the inductance was used in references $[32,33]$ to produce the phase-gradient in the metasurface. Such gradient consisted in continuously increasing the phase of the PRS.

Here, in contrast to previous studies [28, 29, 32, 33], we show that an incrementing phase in the PRS is not necessary to obtain a desired beam steering from the cavity antenna. Instead, we apply phase modulations as illustrated in Figure 2a. The metasurface is considered as a virtual array of antennas closely spaced. By analogy with conventional phased array antennas, a transmission phase difference of $\frac{2 \pi}{\lambda} p \sin \theta$ must be applied between two consecutive cells of the metasurface to achieve beam steering. Also as presented in Figure 2b, the cells are repeated with respect to the center of the cavity as follows: cell $1=$ cell $3=$ cell $5=\ldots$ and cell $2=$ cell $4=$ cell $6=\ldots$. This topology is explained by the fact that the reflection phase difference inside the cavity between two adjacent cells remains the same for the rest of the structure. We take advantage of the phase accumulated by the path length of the wave travelling inside the cavity and the reflections on the ground plane and the PRS cells. Therefore, the transmission phase shift is calculated only for the 4 middle cells of the metasurface (cells $-1,-2,1$ and 2) situated above the primary source. Hence, taking these issues into account, we are able to calculate the transmission phase values of the 4 middle cells in the metasurface in order to obtain the desired steering angle. Three different configurations are considered to numerically study the steering performances of FP cavity antennas. The first one consists in applying a constant phase value along the PRS and the two other configurations make use of a phase-modulated PRS.

Cavity antennas having lateral dimensions $600 \mathrm{~mm} \times$ $600 \mathrm{~mm}$ are designed with a separation distance $h=15 \mathrm{~mm}$, corresponding to $\lambda / 9$ at $2.16 \mathrm{GHz}$ (Figure 2b). In all cases, the primary source is a $31.2 \mathrm{~mm} \times 31.2 \mathrm{~mm}$ microstrip patch antenna with a $4 \mathrm{~mm}$ offset coaxial feed. The simulated reflection coefficients of the three different studied configurations are plotted in Figure 3a. For all the antenna cases, a good matching is observed $\left(S_{11}<-13 \mathrm{~dB}\right)$ around $2.16 \mathrm{GHz}$.

A conventional FP cavity antenna denoted as "Fixed FP", incorporating a constant phase PRS (red trace in Figure 2a) and producing a radiated beam at boresight is designed (Figure $3 b$ ). 
(a)

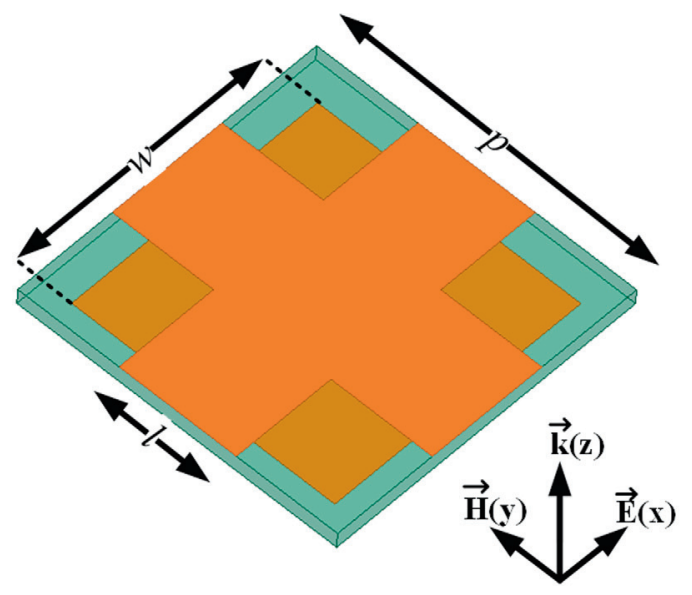

(c)

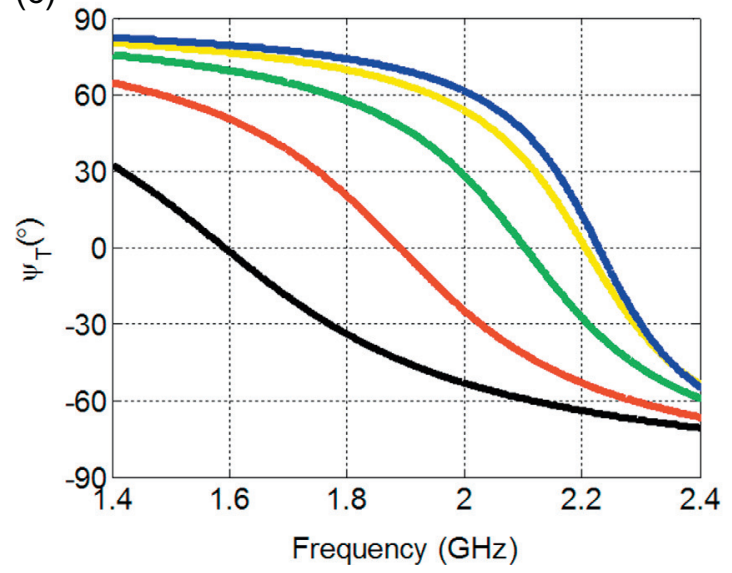

(b)

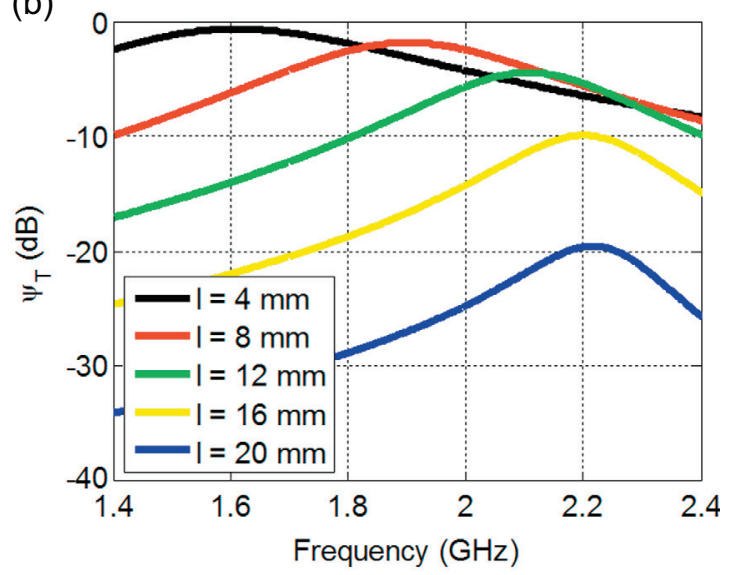

(d)

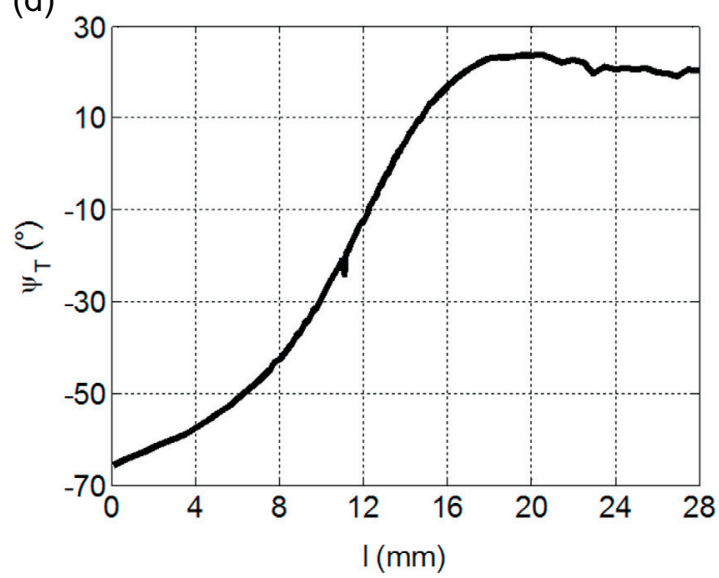

Figure 1. (a) Unit cell of the metasurface composed of an inductive grid and a capacitive grid, (b) transmission magnitude for variable strip width $l$, (c) transmission phase for variable strip width $l$, (d) transmission phase $\psi_{\mathrm{T}}$ that can be achieved along the metasurface by a variation of $l$.

(a)

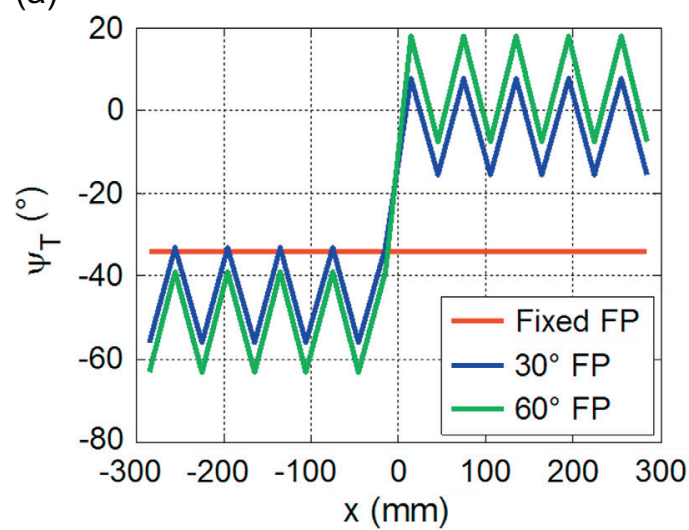

(b)

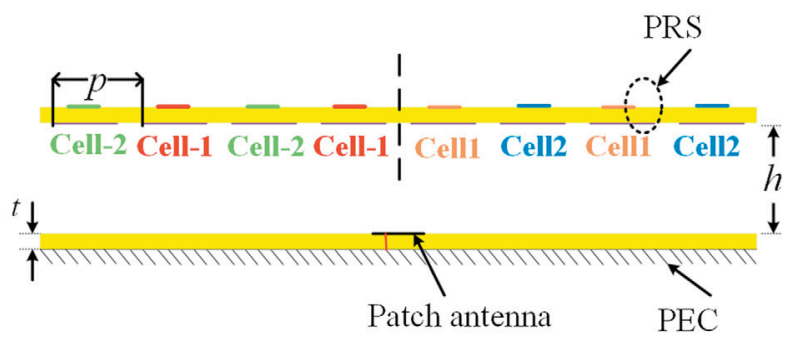

Figure 2. (a) Transmission phase $\psi_{\mathrm{T}}$ along the PRS surfaces for the cavity antennas, (b) schematic view of the cavity composed of a PEC surface and a PRS and embedding a patch antenna as the primary feed.

A non-steered beam is observed due to the uniform PRS presenting a transmission phase of $-34^{\circ}$ along its surface. The dimension $l$ used in the inductive grid is equal to $10.2 \mathrm{~mm}$. To highlight the influence of the phase-modulated
PRS, two other cavity antennas are designed. In the first one referred as " $30^{\circ} \mathrm{FP}$ ", the width $l$ is modulated and is equal to $6.1 \mathrm{~mm}, 9.7 \mathrm{~mm}, 14 \mathrm{~mm}$ and $11.5 \mathrm{~mm}$ on the inductive grid of the PRS. The transmission phase variation of the 
(a)

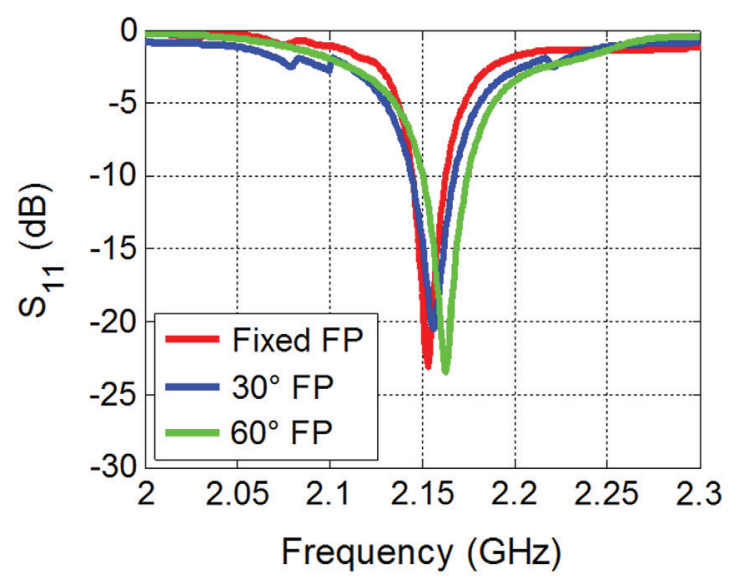

(c)

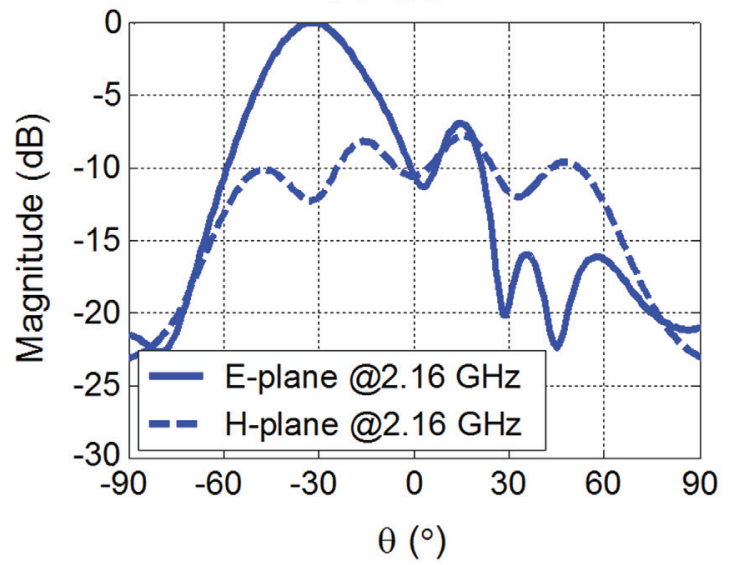

(e)

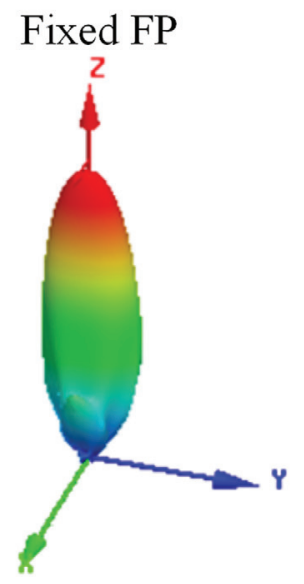

(b)

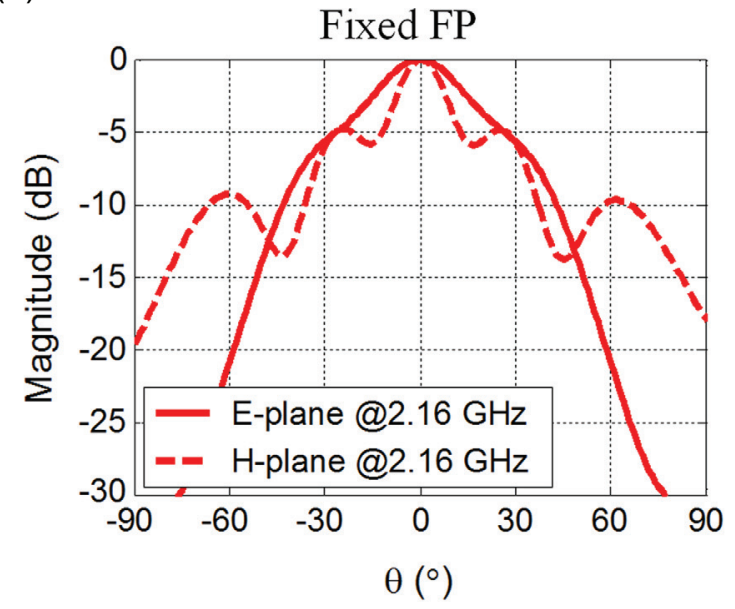

(d)

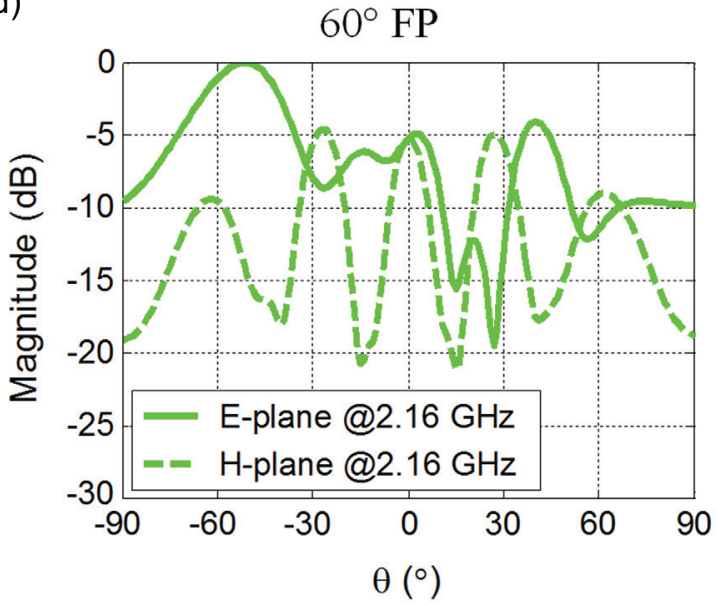

$60^{\circ} \mathrm{FP}$
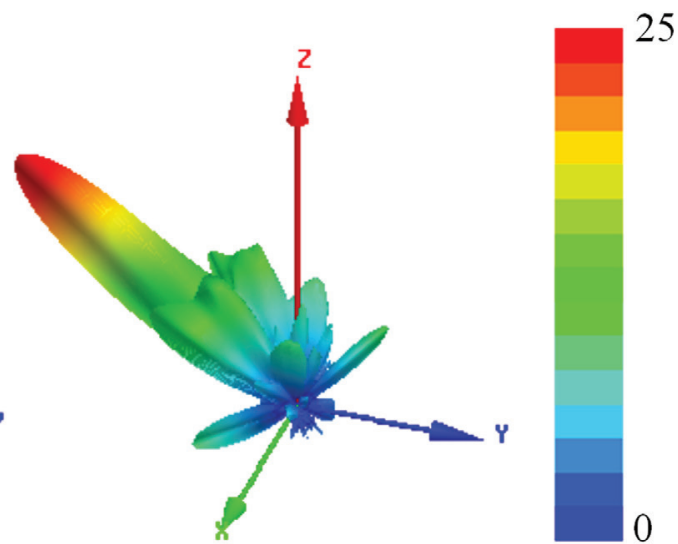

Figure 3. Full-wave simulation results of the different configurations of the Fabry-Perot leaky wave antennas. (a) $S_{11}$ coefficient. (b)-(d) 2D radiation patterns of respectively Fixed FP, $30^{\circ} \mathrm{FP}$ and $60^{\circ} \mathrm{FP}$, (e) 3D radiation patterns (linear scale) of the antennas.

PRS is shown by the blue trace in Figure 2a. The resulting 2D radiation patterns presented in Figure $3 \mathrm{c}$ show a beam steering of $32^{\circ}$ at $2.16 \mathrm{GHz}$. A second configuration denoted " $60^{\circ} \mathrm{FP}$ " where $l$ equal to $4.1 \mathrm{~mm}, 9 \mathrm{~mm}, 15.4 \mathrm{~mm}$ and $12.3 \mathrm{~mm}$ is applied to the PRS, is considered. Therefore a higher phase modulation, as illustrated by the green trace in Figure 2a, has been applied to reach a higher beam steering. Numerical results show a beam steering of $52^{\circ}$ at $2.16 \mathrm{GHz}$ (Figure 3d). Figure $3 \mathrm{e}$ shows the simulated $3 \mathrm{D}$ radiation patterns of the considered cavity antennas, where the influence of the 
(a)

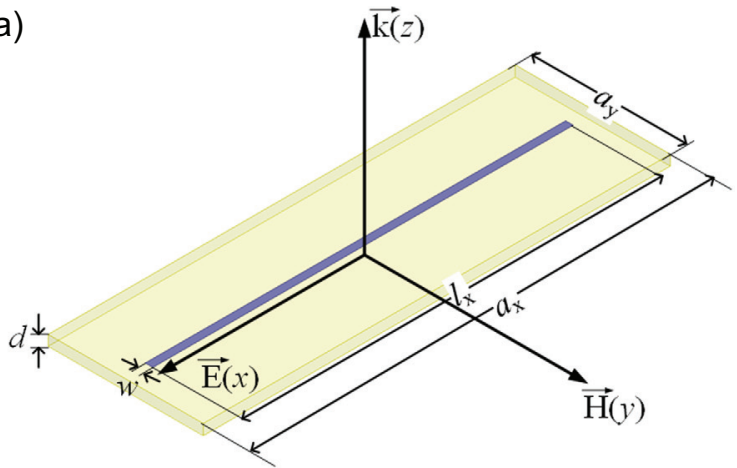

(b)

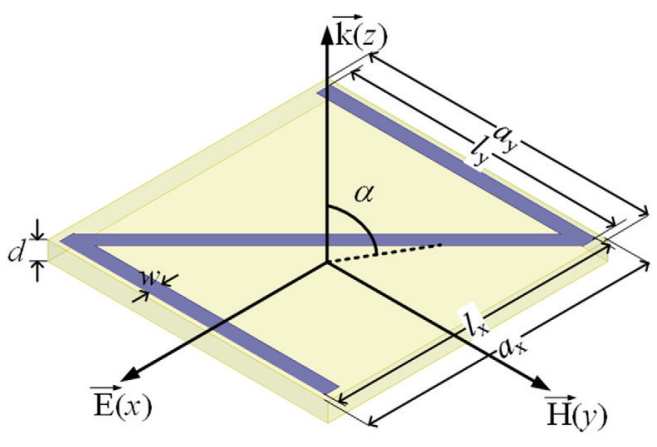

(c)

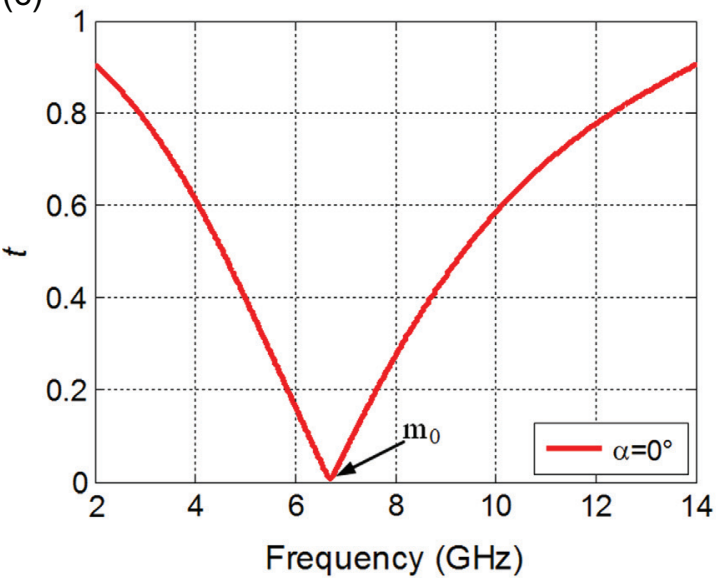

(e) (d)

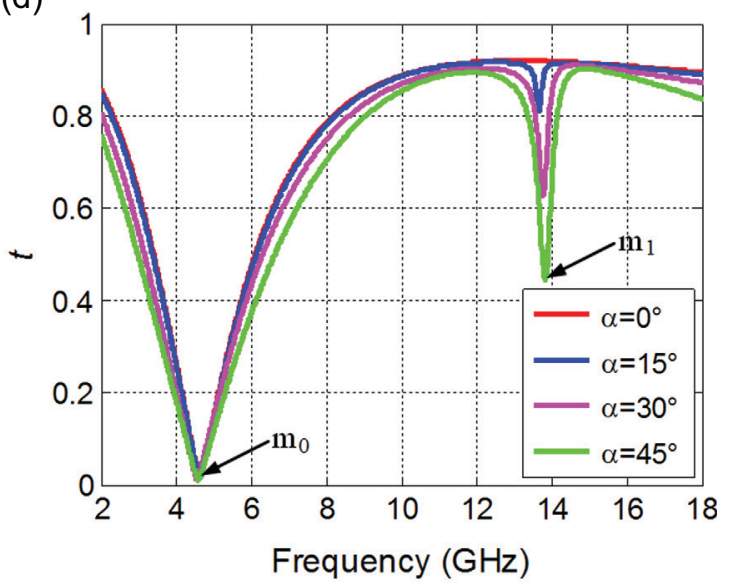

(f)
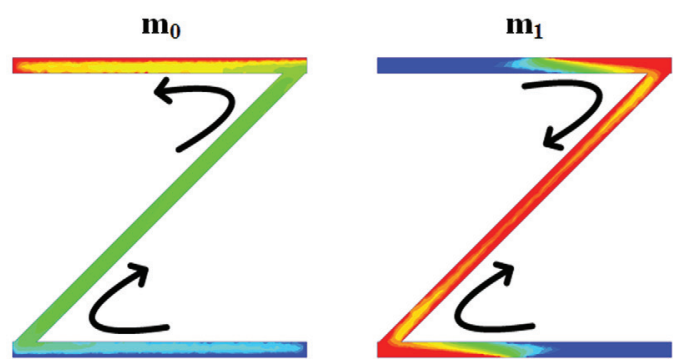

Figure 4. (a) Unit cell of the single-layered cut wires metasurface. The geometrical dimensions of the unit cell are: $a_{x}=20 \mathrm{~mm}, a_{y}=6 \mathrm{~mm}$, $l_{x}=19.6 \mathrm{~mm}, w=0.3 \mathrm{~mm}$ and $d=0.4 \mathrm{~mm}$, (b) unit cell of the single-layered $\mathrm{Z}$ metasurface. The geometrical dimensions of the unit cell are: $a_{x}=a_{y}=6 \mathrm{~mm}, l_{x}=5.8 \mathrm{~mm}, l_{y}=5.7 \mathrm{~mm}, w=0.3 \mathrm{~mm}$ and $d=0.4 \mathrm{~mm}$, (c) simulated transmission response of the cut wires metasurface under normal incidence, (d) simulated transmission responses under normal and oblique incidence of the single-layered $\mathrm{Z}$ metasurface in H-plane, (e) charge distribution at the fundamental $\left(m_{0}\right)$ resonance of the cut wires structure. Dark mode is not excited under normal incidence due to a zero net dipolar momentum, (f) charge distribution at the fundamental $\left(m_{0}\right)$ and first higher order $\left(m_{1}\right)$ resonances of the $\mathrm{Z}$ structure. Under normal incidence, there is no creation of dipolar magnetic momentum and therefore dark mode is not excited. However under off-normal incidence, magnetic field excitation occurs and currents flow in opposite direction in the $\mathrm{Z}$ structure, creating a magnetic momentum and exciting the dark mode.

phase-modulated PRS can be clearly observed on the deviation of the radiated beam.

The study made on these antennas allows to show the interesting applications of metasurfaces in the design of highly directive low-profile antennas. Beam steering can also be considered by analogy with classical array antennas. Instead of applying a phase shift between radiating elements, here a phase modulation is applied on the metasurface to create the desired beam steering.

\section{Metasurfaces for sensing}

\subsection{High quality factor resonant metasurface design}

In this section, we present the design of metasurfaces where excitation of dark modes is not based on mode hybridization but rather on symmetry matching conditions. The great sensitivity of mode hybridization with respect to 
(a)

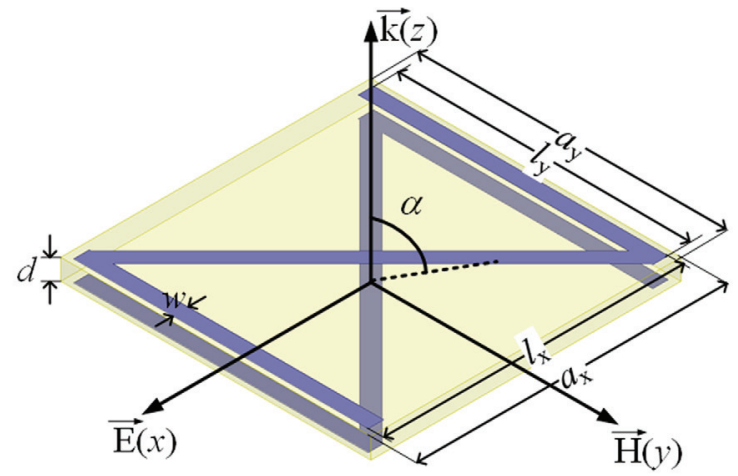

(b)

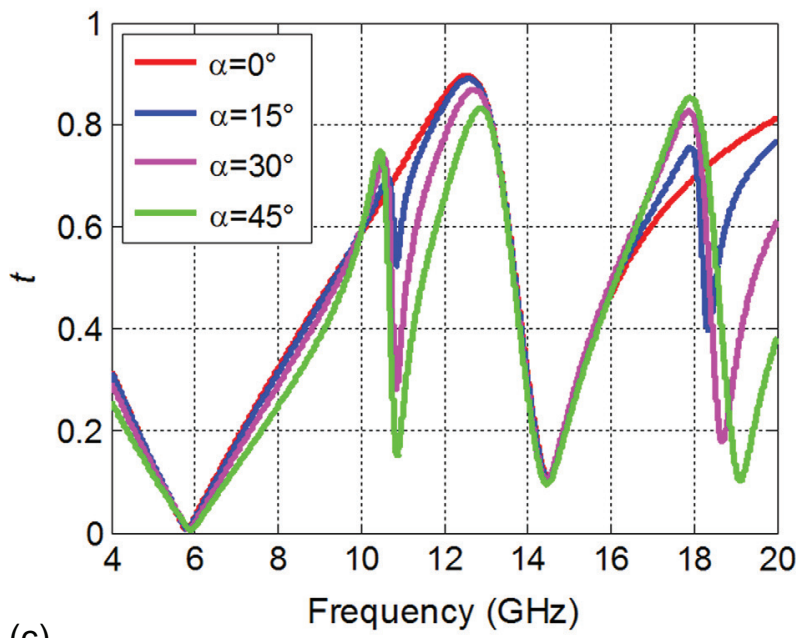

(c)

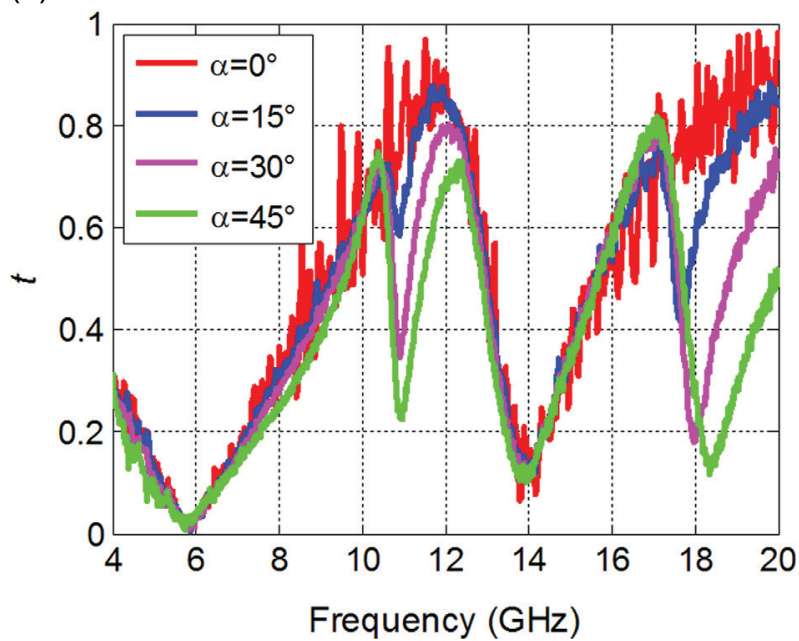

Figure 5. (a) Unit cell and photography of the bi-layered enantiomeric $Z$ metasurface. The geometrical dimensions are the same as for the single-layered $\mathrm{Z}$ structure, (b) simulated transmission responses under normal and oblique incidence in H-plane, (c) measured transmission responses.

the variation of inter-element separation distance makes the reliable fabrication of such structures in the optical domain highly challenging. Therefore, an absence of hybridization mechanism allows to considerably relax the tolerances for the fabrication of such nanostructures at optical frequencies.

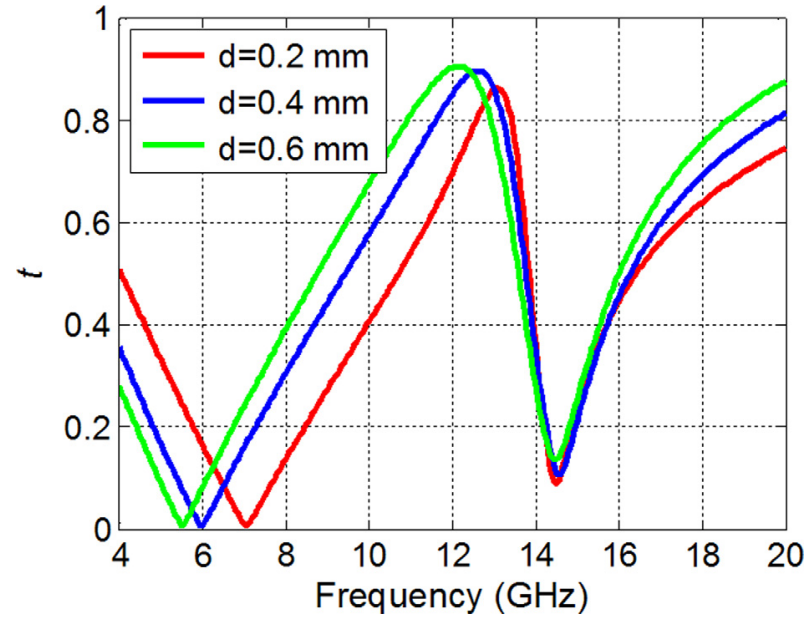

Figure 6. Calculated transmission coefficients under normal incidence for bi-layered enantiomeric metamaterial with different dielectric spacer thickness $d$. The second higher order electric mode resonance frequency is insensitive to the variation of $d$ conversely to the $L C$ fundamental mode.

The main goal here is therefore to present a single structure topology that enables the excitation of dark modes without the need of hybridization between two or three different structures.

To present our approach, we consider firstly a cut wires (CW) based metasurface where a uniform electromagnetic plane wave is normally incident, as presented in Figure 4a. A transmission dip related to the electric dipole mode excitation is observed (Figure 4c). It represents the lowest eigenmode frequency where current flows from one end to the other, as illustrated in Figure 4e. Besides this bright mode, the $\mathrm{CW}$ structure also possesses dark eigenmodes that do not manifest in the spectral response because of the zero net dipolar momentum.

We consider therefore an odd symmetry structure composed of a Z-shaped meta-atom shown in Figure 4b. From considerations related to the symmetry of a structure with respect to the center of the unit cell, it follows that the odd geometry of the $\mathrm{Z}$ structure allows avoiding coupling with a uniform electric field [34]. The total length of the $\mathrm{Z}$ element is $16.3 \mathrm{~mm}$, with $l_{x}=5.8 \mathrm{~mm}, l_{y}=5.7 \mathrm{~mm}, w=0.3 \mathrm{~mm}$ and periods $a_{x}=a_{y}=6 \mathrm{~mm}$. The dielectric substrate used is a $0.4 \mathrm{~mm}$ thick copper-cladded epoxy with a relative dielectric permittivity of 3.9 with tangential losses of 0.02 . As presented in Figure 4d, the calculated transmission spectrum of the single $\mathrm{Z}$ layer structure with the electric field directed along the $x$-direction shows a resonance around $4.6 \mathrm{GHz}$ for all incidence angles. This resonance corresponds to the lowest inductor-capacitor $(L C)$ resonance frequency of the Z-shaped meta-atom. The resonance quality factor, which is given as the ratio of resonant frequency $f_{\mathrm{r}}$ to half-power frequency bandwidth $\Delta f\left(Q=f_{\mathrm{r}} / \Delta f\right)$, is very low $\left(Q_{0}=0.7\right)$ because of the strong radiative coupling to free space. Under normal incidence, because of the even symmetry of the electric field and odd dark mode symmetry, the resulting interaction overlap is null and therefore dark mode is not excited. 
(a)

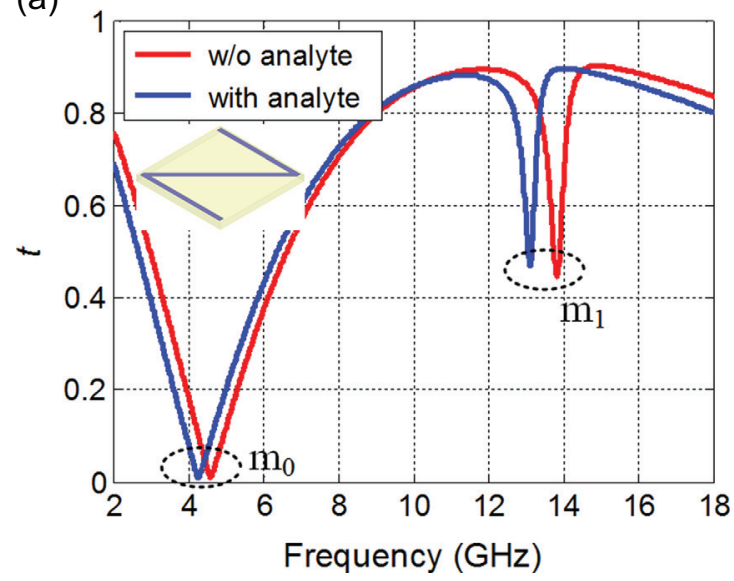

(b)

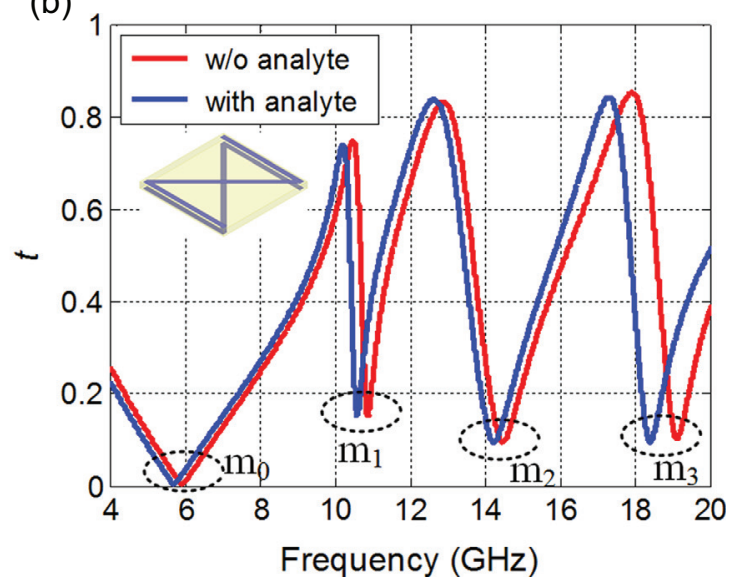

Figure 7. Calculated transmission coefficients under $45^{\circ}$ oblique incidence with and without analyte (thickness $=50 \mu \mathrm{m}$ and dielectric constant $=3$ ). (a) single-layered $\mathrm{Z}$ structure, (b) bi-layered enantiomeric structure.

To excite the dark mode, we make use of the magnetic component of the incident field [35-37]. As the magnetic field transforms as a pseudovector, its symmetry is odd. The direct dark mode excitation is thus allowed for an oblique incidence. Therefore for off-normal incidence in the H-plane, with the electric field still vertically aligned along the $x$-direction, the excitation of an additional resonance is obtained around $13.8 \mathrm{GHz}$. It manifests as the appearance of a narrow reflection frequency band. Even though dark mode excitation is achieved through a direct field coupling, the resonance quality factor of this latter mode $\left(Q_{1}=19\right)$ is much higher than the fundamental one. This is due to the smaller radiative damping corresponding to that of a magnetic dipole.

The dark mode excitation corresponds to the generation of currents flowing in the same clockwise or counterclockwise direction and results in a net dipolar magnetic momentum. It follows that an external magnetic field can thus directly feed the charge displacement corresponding to the resonant dark mode excitation. For this dark mode resonance, due to the opposite direction of the currents flow in the adjacent $\mathrm{Z}$ legs, the accumulated charges are of the same sign. The oppositely directed currents flow in the adjacent $Z$ legs create an additional loop making the dark mode excitation more efficient, as illustrated in Figure 4f.

In order to excite dark mode resonances under normal incidence, a bi-layered enantiomeric metasurface composed of two anti-symmetric stacked $\mathrm{Z}$ structures is considered. The unit cell of the metasurface is shown in Figure 5a. Conversely to the single-layered $\mathrm{Z}$ structure which does not present any signature for the excitation of the second higher order mode, the bi-layered one shows an efficient excitation of the second higher order mode at $14.5 \mathrm{GHz}$, as presented in Figure $5 \mathrm{~b}$. The main advantage of the enantiomeric structure is that it possesses both even and odd symmetry properties. It is therefore possible to excite the second high order electric mode by the interaction of the magnetic momenta formed by current loops, which are induced by external electric field, between the horizontal arms of the $\mathrm{Z}$ meta-atoms.
The evidence of the direct excitation mechanism is provided in Figure 6, where the thickness $d$ of the dielectric substrate separating the two $\mathrm{Z}$ meta-atoms is varied in the bi-layered enantiomeric metasurface. As it can be clearly noted, the fundamental resonance at $6 \mathrm{GHz}$ is only slightly sensitive to the variation of coupling strength controlled via the thickness $d$ of dielectric spacer. The slight shift to lower frequencies for higher separation $d$ is due to the increase of inductance when current loops become bigger. However, the frequency of the dark mode resonance at $14.5 \mathrm{GHz}$ is insensitive to the variation of $d$, indicating that such mode is not based on the near-field coupling between the two $\mathrm{Z}$ designs.

Under oblique incidence, the bi-layered enantiomeric metasurface supports not only direct excitation of dipolar magnetic mode resonance at $10.7 \mathrm{GHz}$, as in the case of the single-layered metasurface, but also a quadrupolar magnetic mode resonance at $18.3 \mathrm{GHz}$ as shown in Figures $5 \mathrm{~b}$ and $5 \mathrm{c}$ in simulations and measurements, respectively. These magnetic modes possess a zero net electric dipolar momentum leading to less radiation losses and therefore a higher resonance quality factor of the response with respect to the electric dipolar modes. Quality factor for dipolar magnetic resonance at $10.7 \mathrm{GHz}$ is equal to 5.4 in comparison to 0.8 and 3.7 for fundamental and second high order electric modes respectively.

\subsection{Sensing applications}

To verify the sensing mechanism in this work, we performed simulations of the single-layered and bi-layered $\mathrm{Z}$ metasurfaces in the presence of a substance placed above the designs [38]. Figure 7 shows the influence of a $50 \mu \mathrm{m}$ thick analyte having dielectric constant equal to $3\left(n_{\text {analyte }}=1.73\right)$. It is clear that the presence of such a material with refractive index greater than 1 causes the different resonances of the metasurfaces to shift towards lower frequencies. In order to quantify the sensitivity of the metasurfaces, the impact of the analyte thickness and refractive index on the performances of the metasurfaces is studied. The resulting characteristics are 


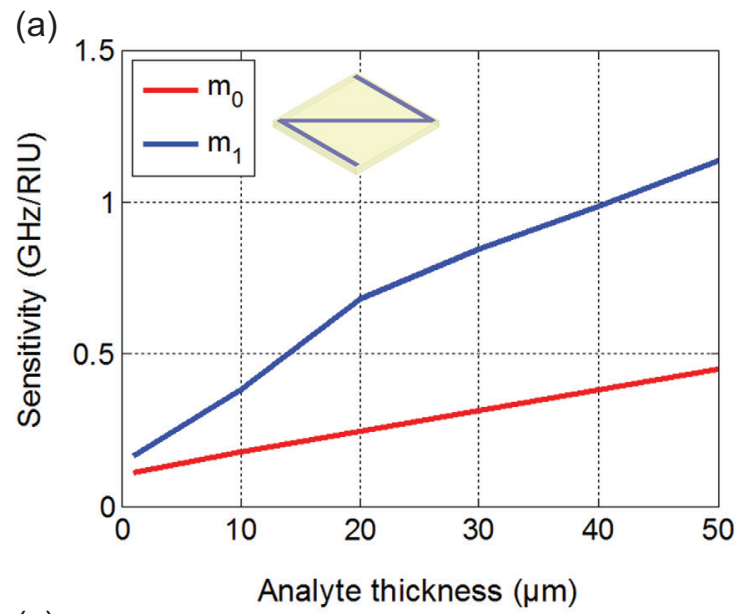

(c)
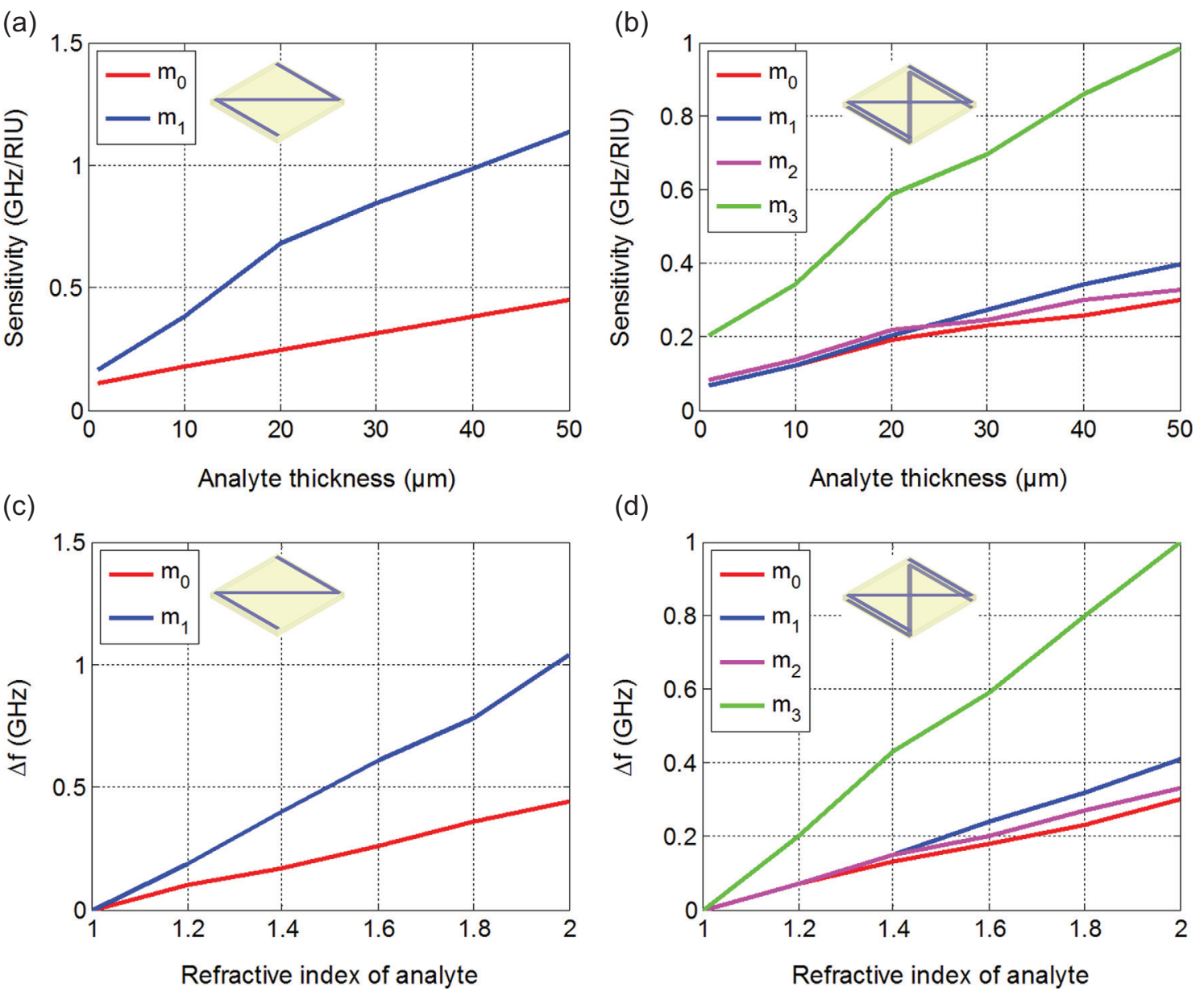

(d)

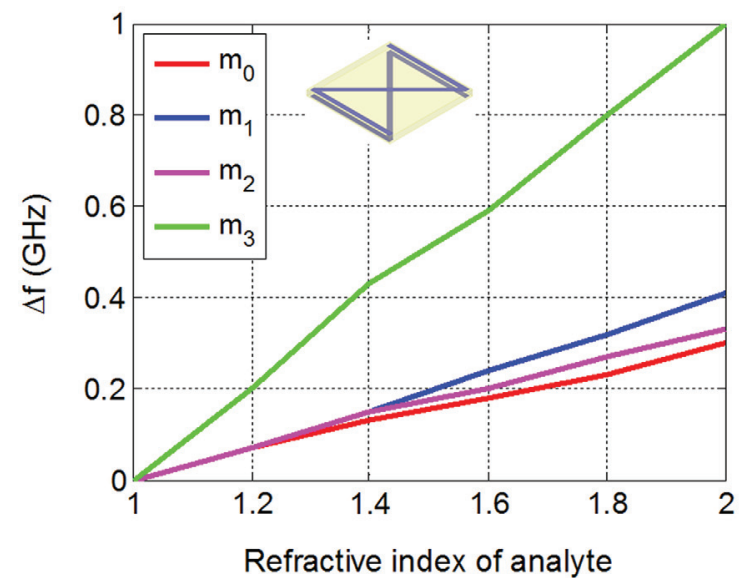

Figure 8. Sensitivity of the metasurfaces with respect to the analyte thickness under $45^{\circ}$ oblique incidence: (a) Single-layered metasurface, (b) bi-layered enantiomeric structure. Sensitivity of the metasurfaces with respect to the analyte refractive index: (c) single-layered metasurface, (d) bi-layered enantiomeric structure.

reported in Figure 8. When the analyte thickness is increased from 1 to $50 \mu \mathrm{m}$, the sensitivity given as the frequency shift $\Delta f$ per refractive index unit (RIU) also increases in both metasurfaces. The excited dark modes are more sensitive to the presence of the substance under test than the fundamental resonance mode. Furthermore, it can be noted the magnetic modes, i.e. dipolar resonance $m_{1}$ in single-layered metasurface and dipolar and quadrupolar resonances $m_{1}$ and $m_{3}$ in bi-layered metasurface, present a higher sensitivity than the electric resonance modes $m_{0}$ and $m_{2}$, particularly for thickness greater than $20 \mu \mathrm{m}$, as presented in Figures $8 \mathrm{a}$ and $8 \mathrm{~b}$.

When the refractive index of a $50 \mu \mathrm{m}$ thick analyte is increased, the frequency shift of the different resonances of the $\mathrm{Z}$ metasurfaces also increases. A frequency shift of more than $1 \mathrm{GHz}$ is obtained from the magnetic resonance mode $m_{1}$ in the single-layered metasurface and from the quadrupolar magnetic resonance mode $m_{3}$ in the bi-layered metasurface. The higher quality factor of the magnetic resonances make these modes more sensitive to the presence of the substance. These results show that the engineered resonances through symmetry matching conditions in the $\mathrm{Z}$ metasurfaces present high sensitivity for the detection of substances.
Therefore such metasurfaces can be considered as good candidates for sensors.

\section{Conclusions}

In summary, the design and engineering of metasurfaces for applications in antenna and sensing domains have been presented. An $L C$-resonant metasurface with high reflectivity was utilized as a partially reflective surface in a Fabry-Perot cavity antenna for high directivity. Applying a phase modulation by variation of the geometrical parameters of the cells composing the metasurface allow to steer the radiated beam to an off-normal direction. Another metasurface composed of only one type of meta-atoms has been exploited to excite dark mode resonances through direct coupling with incident far field rather than the classical hybridization mechanism based on near field coupling between two meta-atoms having different resonance frequencies. Such metasurface has been tested as a sensor in the presence of a substance to be analyzed. A high sensitivity has been observed particularly for the excited magnetic resonance modes due to their higher quality factor since radiative damping is lower. 


\section{Implications and influences}

The results provide a method for designing metasurfaces for high directivity antenna and sensing applications. Though results have been presented in the microwave domain, the dimensions of the constituting cells of the metasurfaces can be easily downscaled for higher frequency applications. Millimeter-wave steered beam antennas and terahertz sensors can therefore be considered.

Acknowledgements. B. Ratni acknowledges partial support of his $\mathrm{PhD}$ works from Airbus Group Innovations. E. Bochkova acknowledges financial support for her PhD scholarship from the French Ministry of Higher Education and Research.

\section{References}

1. F. Falcone, T. Lopetegi, M.A.G. Laso, J.D. Baena, J. Bonache, M. Beruete, R. Marqués, F. Martín, M. Sorolla, Babinet principle applied to the design of metasurfaces and metamaterials, Phys. Rev. Lett. 93 (2004) 197401.

2. E. Saenz, I. Ederra, R. Gonzalo, S. Pivnenko, O. Breinbjerg, P. de Maagt, Coupling reduction between dipole antenna elements by using a planar meta-surface, IEEE Trans. Antennas Propag. 57 (2009) 383-394.

3. A. Dhouibi, S.N. Burokur, A. de Lustrac, A. Priou, Compact metamaterial-based substrate-integrated luneburg lens antenna, IEEE Antennas Wireless Propag. Lett. 11 (2012) 1504-1507.

4. A. Dhouibi, S.N. Burokur, A. de Lustrac, A. Priou, Low-profile substrate-integrated lens antenna using metamaterials, IEEE Antennas Wireless Propag. Lett. 12 (2013) 43-46.

5. S. Maci, G. Minatti, M. Casaletti, M. Bosiljevac, Metasurfing: addressing waves on impenetrable metasurfaces, IEEE Antennas Wireless Propag. Lett. 10 (2011) 1499-1502.

6. A.P. Feresidis, G. Goussetis, S. Wang, J.C. Vardaxoglou, Artificial magnetic conductor surfaces and their application to low-profile high-gain planar antennas, IEEE Trans. Antennas Propag. 53 (2005) 209-215.

7. L. Zhou, H. Li, Y. Qin, Z. Wei, C.T. Chan, Directive emissions from subwavelength metamaterial-based cavities, Appl. Phys. Lett. 86 (2005) 101101.

8. A. Ourir, A. de Lustrac, J.-M. Lourtioz, All-metamaterial-based sub-wavelength cavities $(\lambda / 60)$ for ultrathin directive antennas, Appl. Phys. Lett. 88 (2006) 084103.

9. B. Gallinet, O.J.F. Martin, Refractive index sensing with subradiant modes: a framework to reduce losses in plasmonic nanostructures, ACS Nano 7 (2013) 6978-6987.

10. M.F. Yanik, W. Suh, Z. Wang, S. Fan, Stopping light in a waveguide with an all-optical analog of electromagnetically induced transparency, Phys. Rev. Lett. 93 (2004) 233903.

11. Q. Xu, S. Sandhu, M.L. Povinelli, J. Shakya, S. Fan, M. Lipson, Experimental realization of an on-chip all-optical analogue to electromagnetically induced transparency, Phys. Rev. Lett. 96 (2006) 123901.

12. R.W. Boyd, D.J. Gauthier, Transparency on an optical chip, Nature 441 (2006) 701-702.

13. P. Chak, J.K.S. Poon, A. Yariv, Optical bright and dark states in side-coupled resonator structures, Opt. Lett. 32 (2007) 1785-1787.

14. H. Benisty, Dark modes, slow modes, and coupling in multimode systems, J. Opt. Soc. Am. B 26 (2009) 718-724.
15. B. Luk'yanchuk, N.I. Zheludev, S.A. Maier, N.J. Halas, P. Nordlander, H. Giessen, C.T. Chong, The Fano resonance in plasmonic nanostructures and metamaterials, Nat. Mater. 9 (2010) 707-715.

16. B. Gallinet, O.J.F. Martin, Influence of electromagnetic interactions on the line shape of plasmonic Fano resonances, ACS Nano 5 (2011) 8999-9008.

17. V.A. Fedotov, M. Rose, S.L. Prosvirnin, N. Papasimakis, N.I. Zheludev, Sharp trapped-mode resonances in planar metamaterials with a broken structural symmetry, Phys. Rev. Lett. 99 (2007) 147401.

18. P. Tassin, L. Zhang, T. Koschny, E.N. Economou, C.M. Soukoulis, Low-loss metamaterials based on classical electromagnetically induced transparency, Phys. Rev. Lett. 102 (2009) 053901.

19. R. Singh, C. Rockstuhl, F. Lederer, W. Zhang, Coupling between a dark and a bright eigenmode in a terahertz metamaterial, Phys. Rev. B 79 (2009) 085111.

20. R. Singh, I.A.I. Al-Naib, Y. Yang, D.R. Chowdhury, W. Cao, C. Rockstuhl, T. Ozaki, R. Morandotti, W. Zhang, Observing metamaterial induced transparency in individual Fano resonators with broken symmetry, Appl. Phys. Lett. 99 (2011) 201107.

21. V. Tuz, D. Novitsky, P. Mladyonov, S. Prosvirnin, A. Novitsky, Nonlinear interaction of two trapped-mode resonances in a bilayer fish-scale metamaterial, J. Opt. Soc. Am. B 31 (2014) 2095-2103.

22. A. Christ, Y. Ekinci, H.H. Solak, N.A. Gippius, S.G. Tikhodeev, O.J.F. Martin, Controlling the Fano interference in a plasmonic lattice, Phys. Rev. B 76 (2007) 201405 (R).

23. A. Christ, O.J.F. Martin, Y. Ekinci, N.A. Gippius, S.G. Tikhodeev, Symmetry breaking in a plasmonic metamaterial at optical wavelength, Nano Lett. 8 (2008) 2171-2175.

24. C. Forestiere, L. Dal Negro, G. Miano, Theory of coupled plasmon modes and Fano-like resonances in subwavelength metal structures, Phys. Rev. B 88 (2013) 155411.

25. A. Lovera, B. Gallinet, P. Nordlander, O.J.F. Martin, Mechanisms of Fano resonances in coupled plasmonic systems, ACS Nano 7 (2013) 4527.

26. B. Hopkins, A.N. Poddubny, A.E. Miroshnichenko, Y.S. Kivshar, Revisiting the physics of Fano resonances for nanoparticle oligomers, Phys. Rev. A 88 (2013) 053819.

27. ANSYS HFSS (High Frequency Structure Simulator), version 15, (2012).

28. A. Ourir, S.N. Burokur, A. de Lustrac, Phase-varying metamaterial for compact steerable directive antennas, Electron. Lett. 43 (2007) 493-494.

29. A. Ourir, S.N. Burokur, R. Yahiaoui, A. de Lustrac, Directive metamaterial-based subwavelength resonant cavity antennas - applications for beam steering, Comptes Rendus Phys. 10 (2009) 414-422.

30. R. Guzman-Quiros, J.L. Gomez-Tornero, A.R. Weily, Y.J. Guo, Electronic full-space scanning with 1-D Fabry-Pérot LWA using electromagnetic band-gap, IEEE Antennas Wireless Propag. Lett. 11 (2012) 1426-1429.

31. R. Guzman-Quiros, J.L. Gomez-Tornero, A.R. Weily, Y.J. Guo, Electronically steerable 1-D Fabry-Perot leaky-wave antenna employing a tunable high impedance surface, IEEE Trans. Antennas Propag. 60 (2012) 5046-5055.

32. A. Ghasemi, S.N. Burokur, A. Dhouibi, A. de Lustrac, High beam steering in Fabry-Pérot leaky-wave antennas, IEEE Antennas Wireless Propag. Lett. 12 (2013) 261-264. 
33. A. Ghasemi, S.N. Burokur, A. Dhouibi, A. de Lustrac, Inductive-varying grid for highly beam-steerable cavity antennas, Electron. Lett. 49 (2013) 319-321.

34. W. Zhang, B. Gallinet, O.J.F. Martin, Symmetry and selection rules for localized surface plasmon resonances in nanostructures, Phys. Rev. B 81 (2010) 233407.

35. A. Dhouibi, S.N. Burokur, A. Lupu, A. de Lustrac, A. Priou, "Excitation of trapped modes from a metasurface composed of only Z-shaped meta-atoms, Appl. Phys. Lett. 103 (2013) 184103 .
36. S.N. Burokur, A. Lupu, A. de Lustrac, Direct dark mode excitation by symmetry matching of a single-particle-based metasurface, Phys. Rev. B 91 (2015) 035104.

37. E. Bochkova, S.N. Burokur, A. de Lustrac, A. Lupu, Direct dark modes excitation in bi-layered enantiomeric atoms-based metasurface through symmetry matching, Opt. Lett. 41 (2016) 412-415.

38. R. Yahiaoui, S. Tan, L. Cong, R. Singh, F. Yan, W. Zhang, Multispectral terahertz sensing with highly flexible ultrathin metamaterial absorber, J. Appl. Phys 118 (2015) 083103.

Cite this article as: Ratni B, Bochkova E, Piau G-P, de Lustrac A, Lupu A \& Burokur SN: Design and engineering of metasurfaces for high-directivity antenna and sensing applications. EPJ Appl. Metamat. 2016, 3, 4. 